\title{
Checklist de briófitas (Antocerotophyta, Bryophyta e Marchantiophyta) do estado de São Paulo
}

\author{
Denilson Fernandes Peralta ${ }^{1,2}$ \& Olga Yano ${ }^{I}$ \\ ${ }^{1}$ Núcleo de Pesquisa em Briologia, Instituto de Botânica, \\ Av. Miguel Stéfano, 3687, Água Funda, CEP 04301-902, São Paulo, SP, Brasil, \\ ${ }^{2}$ Autor para correspondência: Denilson Fernandes Peralta, e-mail:denilsonfp@yahoo.com.br
}

PERALTA, D.F. \& YANO, O. Bryophytes checklist (Antocerotophyta, Bryophyta and Marchantiophyta) of São Paulo State. Biota Neotrop., 11(1a): http://www.biotaneotropica.org.br/v11n1a/en/abstract?inventory+ bn0111101a2011

\begin{abstract}
All publications mentioning samples from state of São Paulo were revised and 10 names of uncertain occurrence were excluded. All taxa included had samples from the state of São Paulo and are deposited in indexed herbaria. We found 1,324 bryophytes species, including nine Antocerotophyta, 740 to Bryophyta and 575 to Marchantiophyta. We checked at last one sample of these 1,000 names and 324 taxa was cited based on literature. One hundred thirty nine of the taxa recognized in this list have a single citation to present knowledge of the species, this is the type locality in the state of São Paulo. Prospects for bryophytes research in the next 10 years are mainly related to training of human resources for the preparation of the state bryophyte flora, providing tools for studies that use the taxonomic unit (species) as an object.
\end{abstract}

Keywords: Bryophytes, plant biodiversity, mosses, liverworts, hornworts.

Number of Species: In the World: 15.100 (Gradstein et al., 2001; Frahm 2003), in Brazil: 3.125 (Yano 1996): 1.521 (Forzza, 2010), estimated at São Paulo State: 1.350, known at São Paulo State: 1.324

PERALTA, D.F. \& YANO, O. Checklist de briófitas (Antocerotophyta, Bryophyta e Marchantiophyta) do Estado de São Paulo. Biota Neotrop., 11(1a): http://www.biotaneotropica.org.br/v11n1a/pt/abstract?inventory +bn0111101a2011

Resumo: Foram revisadas todas as publicações que citam amostras do estado de São Paulo e excluídos 10 nomes de ocorrência incerta. Foram incluídos os táxons que possuíam amostras provenientes do estado de São Paulo que e apresentavam espécimes depositadas em herbários indexados. Foram encontradas 1.324 espécies de briófitas incluindo nove em Antocerotophyta (9), Bryophyta (740) e Marchantiophyta (575). Foram conferidas as identificações de 1.000 dos táxons citados e 324 baseando-se em literatura. Dos táxons reconhecidos nesta listagem 139 apresentam como única citação para o conhecimento científico da espécie a localidade-tipo dentro do estado de São Paulo. As perspectivas de pesquisa para as briófitas nos próximos 10 anos estão principalmente relacionadas a formação de recursos humanos para a confecção da flora de briófitas do estado, proporcionando assim uma ferramenta indispensável para a realização dos estudos que utilizam a unidade taxonômica (espécie) como objeto para inúmeros trabalhos.

Palavras-chave: Briófitas, biodiversidade de plantas, musgos, hepáticas, antóceros.

Número de espécies: No mundo: 15.100 (Gradstein et al., 2001; Frahm, 2003), no Brasil: 3.125 (Yano 1996): 1.521 (Forzza, 2010), estimadas no estado de São Paulo: 1.350, conhecidas no estado de São Paulo: 1.324 


\section{Introdução}

As briófitas são representadas por três divisões: antóceros (Anthocerotophyta) (Renzaglia et al. 2008), hepáticas (Hepatophyta) (Crandall-Stotler et al. 2008) e musgos (Bryophyta) (Goffinet et al. 2008).

Existem cerca de 15.100 espécies de briófitas no mundo, das quais 10.000 são musgos, 5.000 hepáticas e 100 antóceros (Gradstein et al. 2001, Frahm 2003). Para o Brasil já foram listadas, principalmente nos catálogos de Yano (1981a, 1984a, 1989, 1995, 2006, 2008 e 2010), 3.125 táxons de briófitas, estes catálogos foram compilados em um checklist e sumarizados Yano (1996).

Esta estimativa para o Brasil baseia-se em nomes citados em publicações e herbários, com alguns nomes que devem ser sinonimizados ou revistos, dessa maneira a listagem apresentada em Forzza et al. (2010) são considerados 1.525 táxons de briófitas no catálogo das espécies de Fungos e plantas do Brasil. Este número também é uma aproximação, haja visto que a metodologia utilizada pelo grupo das briófitas utilizou toda a literatura disponível atualizando vários nomes considerados sinônimos e mesmo assim alguns nomes não puderam ser resolvidos, como aqueles conhecidos apenas pelo material-tipo e que não possuem revisão recente.

Cerca de $20 \%$ dos táxons citados para o Brasil já foram revisados taxonomicamente. Porém, a estimativa de Yano (1996) parece ser o número provável de espécies que ocorrem no Brasil (Shepherd 2003). Neste caso, o Brasil possui aproximadamente $20 \%$ de todas as briófitas conhecidas no mundo.

O estado de São Paulo apresenta extenso histórico de exploração das briófitas, principalmente relacionado a coleta de espécimes, o herbário do Estado "Maria Eneyda P. Kauffmann Fidalgo" (SP) possui cerca de 85.000 exemplares de briófitas, e a metade desse acervo é proveniente do estado de São Paulo foi coletado pelo Ms. Daniel Moreira Vital e pela Dra. Olga Yano. Juntos estes pesquisadores também são responsáveis por inúmeros trabalhos sobre as briófitas do estado de São Paulo.

Existe para o Brasil cerca de 1.150 trabalhos que listam espécies de briófitas, a Dra. O. Yano realizou grande esforço ao sumarizar estes trabalhos nos catálogos de Yano (1981a, 1984a, 1989, 1995, 2006, 2008 e 2010) e estes trabalhos são sempre utilizados em levantamento, e não existe necessidade de listá-los novamente e serão apresentados apenas os trabalhos históricos mais importantes e recentes que abrangeram grande número de espécies realizados apenas para o estado.

Yano \& Bastos (1998) apresentam um histórico dos estudos com briófitas e entre os trabalhos citados destacam-se Puiggari (1881) e Loefgren (1896), por serem os pioneiros e mais recentemente os trabalhos de Hell (1969), Yano (1975), Vital (1980), Visnadi (1998) e Peralta \& Yano (2010) que trataram de grande quantidade de táxons.

\section{Metodologia}

Para a confecção da listagem dos táxons de briófitas ocorrentes no estado de São Paulo foram consultadas as bibliografias citadas na introdução e, principalmente os trabalhos de revisão taxonômica.

Foram incluídos apenas os táxons que possuíam amostras provenientes do estado de São Paulo e apresentavam espécimes depositadas em herbários indexados.

Foram revisadas todas as publicações que citam amostras do estado de São Paulo e excluídos cerca de 10 nomes que apresentam citação de ocorrência dentro do estado, porém não apresentavam citação de material examinado, além destes táxons não serem reencontrados com identificações recentes.

Foram conferidas as identificações de 1.000 dos táxons citados aqui (aqueles que apresentavam espécimes depositados no Herbário
SP), 13 táxons apresentavam revisões recentes e 311 foram incluídos exclusivamente baseando-se em literatura.

Os nomes listados foram compilados dos trabalhos realizados para o Brasil ou Neotrópico e as famílias que tiveram suas espécies revisadas e, portando com alta confiabilidade taxonômica, foram: Amblystegiaceae (Hëdenas 2003), Aytoniaceae (Yano 1981b), Calymperaceae (Reese 1993), Cryphaeaceae - Cryphaea (Rao 2001), Dicranaceae - Campylopodioideae e Paraleucobryoideae (Frahm 1991), Erpodiaceae (Vital 1980), Fissidentaceae (Pursell 2007), Helicophyllaceae (Yano 1979), Hypnaceae (Ireland \& Buck 2009), Lejeuneaceae - Ceratolejeunea (Dauphin 2003), Lejeuneaceae - Holostipae (Gradstein 1994), Lejeuneaceae - Prionolejeunea (Ilkiu-Borges 2005), Leucobryaceae (Yano 1992), Leucophanaceae (Salazar-Allen 1993), Marchantiaceae - Marchantiidae (BischlerCausse et al. 2005), Metzgeriaceae (Costa 2008), Neckeraceae e Thamnobryaceae (Sastre-de Jesus 1987), Phyllogoniaceae (Yano \& Mello 1989), Pilotrichaceae - Lepidopilum (Churchill 1988), Plagiomniaceae (Yano 1990), Plagiotheciaceae (Buck \& Ireland 1989), Polytrichaceae (Peralta \& Yano 2010). Racopilaceae (Yano 1984b), Rhachitheciaceae (Yano 1985), Rhizogoniaceae (Yano 1986), Ricciaceae - Ricciocarpus (Yano 1981c), Sphagnaceae - Sphagnum (Yano et al. 1985), Stereophyllaceae (Ireland \& Buck 1994).

Além de novas ocorrências apartir de táxons recém identificados pelos autores para o estado que não haviam sido publicados.

\section{Resultados e Discussão}

Estão sendo apresentadas 1.324 espécies de briófitas incluindo nove em Antocerotophyta, 740 em Bryophyta e 575 em Marchantiophyta para o Estado de São Paulo. Este número tem aumentado progressivamente desde a primeira lista para o estado realizada por Yano \& Bastos (1998) (1.156 espécies) e Yano (1998) (1.166 espécies) e já ultrapassou a estimativa apresentado por Yano (1998) de 1.250 espécies.

O trabalho realizado para a compilação dos táxons foi importante para o conhecimento da diversidade do grupo no Estado e através da análise desta lista foi possível saber que alguns nomes são conhecidos apenas do material-tipo e necessitam serem revisados.

Dos táxons reconhecidos nesta listagem 139 apresentam como única citação para o conhecimento científico da espécie a localidadetipo dentro do estado de São Paulo.

É interessante mencionar a espécie Isotachis simonensi Fittipaldi, recentemente descrita em 1990, a partir de material fóssil.

O número de táxons obtido para o estado representa $42 \%$ do número citado por Yano (1998) e 87\% do citado por Forzza et al. (2010). Porém esta comparação é muito imprecisa, uma vez que ainda existem muitas famílias que necessitam ser revisadas e dessa maneira a comparação com os 1.039 táxons citados por Costa et al. (2005) para o estado do Rio de Janeiro fica extremamente inconsistente.

Não é possível analisar a distribuição das briófitas dentro do estado devido a metodologia adotada, porém esta lista fornecerá subsídios para a confecção da flora de briófitas do estado e consequentemente análises fitogeográficas.

A maior representatividade das briófitas para o estado está no herbário do Estado "Maria Eneyda P. Kauffmann Fidalgo" (SP), onde existem espécimes depositados de 1.000 dos táxons citados e o herbário de Viena (W) com 224 táxons.

A coleção de briófitas do herbário SP está $55 \%$ informatizada no programa BRAHMS e toda a inclusão de amostras está sendo realizada diretamente no programa, dessa maneira as novas amostras incluídas já estarão sendo informatizadas e as etiquetas confeccionadas a partir do próprio programa. Este programa é de 
livre acesso, e será utilizado em conjunto com Specieslink (CRIA) para futura disponibilização da coleção na Internet.

A grande representatividade da flora do estado de São Paulo no herbário SP reflete a atividade do grupo de pesquisa de briófitas do Instituto de Botânica, o único local do Brasil onde trabalham três doutores especialistas em briófitas. Os herbários brasileiros com coleções de briófitas foram sumarizados por Costa \& Pôrto (2003) e o herbário SP é o maior do Brasil em espécimes de briófitas.

Uma das maiores prioridades para o conhecimento das briófitas brasileiras é a formação de novos pesquisadores capacitados para coletar, identificar e estudar estas plantas (Shepherd 2003, Costa \& Pôrto 2003, Menezes et al. 2005).

Infelizmente, no estado de São Paulo, apenas a Dra. Olga Yano está vinculada a Programa de Pós-graduação e dessa maneira a formação de recursos humanos está sub-explorada. Recentemente, com o Doutorado de Denilson F. Peralta também está empenhado em formar estudantes.

As principais lacunas no conhecimento das briófitas observados através da elaboração desta listagem encontram-se nas amostras depositadas no herbário de Viena (W) referente as coleções de Puiggari e Schiffner.

A metodologia utilizada não permite avançar, porém através de conhecimento prévio as principais lacunas no conhecimento das briófitas do Estado de São Paulo estão nas famílias: Aneuraceae, Bartramiaceae, Dicranaceae, Hypnaceae, Jubulaceae, Lejeuneaceae, Neckeraceae, Orthotrichaceae, Pilotrichaceae, Plagiochilaceae e Sematophyllaceae. Ou seja, nas famílias com maior número de táxons, para os quais foram descritas muitas espécies em gêneros que não apresentam revisões recentes.

As perspectivas de pesquisa para as briófitas nos próximos 10 anos estão principalmente relacionadas a formação de recursos humanos para a revisão das famílias relacionadas acima para confecção da flora de briófitas do estado, proporcionando assim uma fermenta indispensável para a realização dos estudos que utilizam a unidade taxonômica (espécie) como objeto para inúmeros trabalhos.

\section{Referências Bibliográficas}

BISCHLER-CAUSSE, H., GRADSTEIN, S.R., JOVET-AST, S. LONG, D.G. \& SALAZAR-ALLEN, N. 2005. Marchantiidae.Flora Neotrop. 97:1-262.

BUCK, W.R. \& IRELAND, R.R. 1989.Plagiotheciaceae.Flora Neotrop. 50:1-22.

CHURCHILL, S.P. 1988. A revision of the moss genus Lepidopilum (Callicostaceae).Tese de Doutorado, The City University of New York, New York.

COSTA, D.P. \& PÔRTO, K.C. 2003. Estado da arte das coleções de briófitas no Brasil. In Coleções Biológicas de Apoio ao Inventário, Uso Sustentável e Conservação da Biodiversidade(A.L. Peixoto, org.). Instituto de Pesquisas Jardim Botânico do Rio de Janeiro, Rio de Janeiro,p.75-98.

COSTA, D.P. 2008. Metzgeriaceae (Hepaticae). Flora Neotrop.102:1-169.

COSTA, D.P., IMBASSAHY, C.A.A. \& SILVA, V.P.A.V. 2005. Checklist and distribution of mosses, liverworts and hornworts of Rio de Janeiro State, Brazil.J. Hattori Bot.1 Lab.98:259-298. http://dx.doi.org/10.1590/ S1679-87592006000300006

CRANDALL-STOTLER, B., STOTLER, R.E. \& LONG, D.G. 2008. Morphology and classification of the Marchantiophyta.In Bryophyte Biology(B. Goffinet\& A.J. Shaw).2nd ed.Cambdrige University Press,p.1-54.

DAUPHIN, L.G. 2003. Ceratolejeunea (Lejeuneaceae, Lejeuneoideae).Flora Neotrop. 90:1-86.

FORZZA, R.C., org. 2010. Catálogo das Plantas e Fungos do Brasil. Jardim Botânico do Rio de Janeiro,v.1, 875 p.

FRAHM, J.-P. 1991. Dicranaceae: Campylopodioideae, Paraleucobryoideae. Flora Neotrop. 54: 1-238.
FRAHM, J.-P., ed. 2003.Manual of Tropical Bryology. Trop. Bryol. 23:1-195.

GOFFINET, B., BUCK,W.R. \& SHAW, A.J. 2008.Morphology, anatomy and classification of the Bryophyta.In Bryophyte Biology(B. Goffinet\& A.J. Shaw).2nd ed. Cambdrige University Press, p.56-138.

GRADSTEIN, S.R., CHURCHILL, S.P. \& SALAZAR-ALLEN, N. 2001. Guide to the Bryophytes to Tropical America. Mem.New York Bot. Gard.86:1-577.

GRADSTEIN, SR. 1994.Lejeuneaceae: Ptychantheae, Brachiolejeuneae. Flora Neotrop. 61:1-216.

HËDENAS, L. 2003. Amblystegiaceae (Musci). Flora Neotrop. 89:1-107.

HELL, K.G. 1969. Briófitas talosas dos arredores da cidade de São Paulo (Brasil). Bol. Fac. Filos., Cienc. Let. Univ. Sao Paulo.,Ser. Bot.25(335):1187.

ILKIU-BORGES, A.L. 2005.A taxonomic monograph of the genus Prionolejeunea (Lejeuneaceae, Jungermanniopsida).CuvillierVerlag, Götting, 191p.

IRELAND, R.R. \& BUCK, W.R. 1994. Stereophyllaceae.Flora Neotrop. 65:1-49.

IRELAND, R.R. \& BUCK, W.R. 2009. Some Latin American genera of Hypnaceae (Musci).Smithson. Contrib. Bot. 93:1-97.http://dx.doi. org/10.5479/si.0081024X.93

LOEFGREN, A. 1896. Índice das plantas do herbário da Comissão Geographica e Geológica de São Paulo. Bol. Com. Geog. Geol. São Paulo, Serv. Met. 11:51-215.

MENEZES, M.M., MAIA, L.C., COSTA, D.P. \& BICUDO, C.E.M. 2005. Coleções de plantas avasculares e fungos como base de conhecimento para a diversidade biológica brasileira: uma reavaliação. CRIA. www. cria.org.br/cgee/col

PERALTA, D.F. \&YANO, O. 2008. Briófitas do Parque Estadual da Ilha Anchieta, Ubatuba, estado de São Paulo. Iheringia, Sér. Bot. 63(1):101127.

PERALTA, D.F. \& YANO, O. 2010. Taxonomic treatment of the Polytrichaceae from Brazil.The Bryologist113(3):646-672.http://dx.doi. org/10.1639/0007-2745-113.3.646

PUIGGARI, J.I. 1881. Notícia sobre algumas criptógamasnuevashalladas em Apiahy, província de San Pablo enel Brasil. An. Soc. Cient. Argentina11:201-216.

PURSELL, R.A. 2007. Fissidentaceae. Flora Neotrop. 101:1-278.

RENZAGLIA, K.S., VILLARREAL, J.C. \& DUFF, R.J. 2008. New insights into morphology, anatomy and systematics of hornworts.In Bryophyte Biology (B. Goffinet\& A.J. Shaw).2nd ed. Cambdrige University Press, p.139-171.

RAO, P. 2001. Monographic studies on Cryphaea (Bryopsida). Bryobrothera 7:1-112.

REESE, W.D. 1993. Calymperaceae.Flora Neotrop. 58:1-102.

SALAZAR-ALLEN, N. 1993.LeucophanaceaeFlora Neotrop. 59:1-11

SASTRE-DE JÉSUS, I. 1987.A revision of the NeckeraceaeSchimp.and the Tamnobryaceae Marg. \&Dur. in the Neotropics. Tese de Doutorado, The City University of New York,New York.

SHEPHERD, G.J. 2003. Plantas terrestres: Versão preliminar. 60 p.Relatório de Avaliação do estado do conhecimento da diversidade biológica do Brasil.

VISNADI, SR. 1998. Briófitas em Ecossitemas Costeiros do Núcleo Picinguaba do Parque Estadual da Serra do Mar, Ubatuba - SP. Tese de Doutorado, Univ. Estadual Paulista Campus de Rio Claro, 274p.

VITAL, D.M. 1980. Erpodiaceae (Musci) do Brasil. Dissertação de Mestrado, Universidade Estadual de Campinas, 135p.

YANO, O. 1975. Leucobryaceae (Musci) do Estado de São Paulo. Dissertação de Mestrado, Escola Paulista de Medicina, Brasil.

YANO, O. 1979. Contribuição ao inventário dos Musci Brasileiros: Helicophyllaceae. Rickia 8:7-16.

YANO, O. 1981a. A Checklist of Brazilian mosses. J. Hattori Bot.1 Lab.50:279456. 
YANO, O. 1981b. Aytoniaceae (Marchantiales, Hepaticopsida) no Brasil. Rev. Bras. Bot. 4(2):89-94.

YANO, O. 1981c. Distribuição de Ricciocarpusnatans (L.) Corda (Marchantiales, Hepaticopsida) no Brasil. Rickia 9:1-5.

YANO, O. 1984a. Checklist of Brazilian liverworts and hornworts.J. Hattori Bot.1 Lab.56:481-548.

YANO, O. 1984b. Contribuição ao Inventário dos Musci Brasileiros: 3. Racopilaceae (Bryopsida, Isobryales). Rev. Bras. Bot. 7(1):57-63.

YANO, O. 1985. Contribuição ao inventário dos Musci Brasileiros: 4. Rhachitheciaceae. Rickia12:29-34.

YANO, O. 1986. Contribuição ao inventário dos Musci Brasileiros: 5. Rhizogoniaceae (Bryopsida). Rickia 13:49-60.

YANO, O. 1989. An additional checklist of Brazilian bryophytes.J. Hattori Bot.1 Lab. 66:371-434.

YANO, O. 1990. Estudos de Briófitas do Brasil: Plagiomniaceae (Bryopsida). Rev. Bras. Bot. 13(2):103-108.

YANO, O. 1992. Leucobryaceae (Bryopsida) do Brasil. Tese de Doutorado, Universidade de São Paulo, São Paulo.

YANO, O. 1995. A new additional annotated cheklist of Brazilian bryophytes.J. Hattori Bot.1 Lab. 78:137-182.
YANO, O. 1996. A checklist of Brazilian bryophytes.Bol. Inst. Bot.10:47-232.

YANO, O. 1998. Briófitas do estado de São Paulo. In Biodiversidade do estado de São Paulo, Brasil - Síntese do conhecimento ao final do século XX 2: Fungos macroscópicos e plantas(C.E.M. Bicudo\& G.I. Shepherd, eds.). São Paulo,v.2, p.37-46.

YANO, O. 2006. Novas adições ao catálogo de briófitas brasileiras. Bol. Inst. Bot.17:1-142.

YANO, O. 2008. Catálogo de Antóceros e Hepáticas brasileiras: literatura original, basiônimo, localidade-tipo e distribuição geográfica. Bol. Inst. Bot.19:1-110

YANO, O. 2010. Levantamento de novas ocorrências de briófitas brasileiras. Instituto de Botânica. CDU582.32/WWW.ibot.sp.gov.br/Briófitas Brasileiras/BriófitasMIOLOECAPA.pdf.

YANO, O., PIRANI, J.R. \& SANTOS, D.P. 1985. O gênero Sphagnum (Bryopsida) nas regiões Sul e Sudeste do Brasil. Rev. Bras. Bot. 8:55-80.

YANO, O. \& BASTOS, C.J.P. 1998. Briófitas do estado de São Paulo, Brasil. In Simpósio de Ecossistemas Brasileiros. ACIESP, v.104, p.200-224.

YANO, O. \& MELLO, Z.R. 1989. Estudos de Briófitas do Brasil: 6. Phyllogoniaceae (Bryopsida). Acta bot. bras. 3(2):119-130.

Recebido em 30/06/2010

Versão Reformulada recebida em 08/10/2010

Publicado em 10/11/2010 